Review Article

\title{
Activating the Antitumor Immune Response in Non-Hodgkin Lymphoma Using Immune Checkpoint Inhibitors
}

\author{
Maansi Joshi and Stephen M. Ansell \\ Division of Hematology, Mayo Clinic, Rochester, MN, USA \\ Correspondence should be addressed to Stephen M. Ansell; ansell.stephen@mayo.edu
}

Received 15 September 2020; Accepted 29 October 2020; Published 19 November 2020

Academic Editor: Lisa Argnani

Copyright (c) 2020 Maansi Joshi and Stephen M. Ansell. This is an open access article distributed under the Creative Commons Attribution License, which permits unrestricted use, distribution, and reproduction in any medium, provided the original work is properly cited.

\begin{abstract}
Non-Hodgkin lymphomas comprise a heterogenous group of disorders which differ in biology. Although response rates are high in some groups, relapsed disease can be difficult to treat, and newer approaches are needed for this patient population. It is increasingly apparent that the immune system plays a significant role in the propagation and survival of malignant cells. Immune checkpoint blocking agents augment cytotoxic activity of the adaptive and innate immune systems and enhance tumor cell killing. Anti-PD-1 and anti-CTLA-4 antibodies have been tested as both single agents and combination therapy. Although success rates with anti-PD-1 antibodies are high in patients with Hodgkin lymphoma, the results are yet to be replicated in those with non-Hodgkin lymphomas. Some lymphoma histologies, such as primary mediastinal B cell lymphoma (PMBL), central nervous system, and testicular lymphomas and gray zone lymphoma, respond favorably to PD-1 blockade, but the response rates in most lymphoma subtypes are low. Other agents including those targeting the adaptive immune system such as TIM-3, TIGIT, and BTLA and innate immune system such as CD47 and KIR are therefore in trials to test alternative ways to activate the immune system. Patient selection based on tumor biology is likely to be a determining factor in treatment response in patients, and further research exploring optimal patient populations, newer targets, and combination therapy as well as identifying biomarkers is needed.
\end{abstract}

\section{Introduction}

Immune therapies have changed the paradigm of cancer treatment, particularly Hodgkin and non-Hodgkin lymphomas. Lymphoma cells, being a part of the immune system, are themselves immunologically active and modulate the host immune response to allow growth of the malignant cell. In addition, the tumor microenvironment (TME) is now being increasingly recognized for its role in immune suppression and propagating tumor growth. Interactions between lymphoma cells and the TME influence $\mathrm{T}$ cell function are crucial for tumor progression. Checkpoint proteins act as natural regulators of $\mathrm{T}$ cell function and help to modulate the $\mathrm{T}$ cell response by creating a balance between activation and inhibition [1].

Cytotoxic T lymphocyte antigen 4 (CTLA-4/CD152) and programmed cell death protein 1 (PD-1/CD279) of the B7 family, among others, are inhibitory molecules which result in reduced $\mathrm{T}$ cell activity and function. Disease tolerance seen in malignancy can be attributed in part to sustained interaction of these proteins with their corresponding ligands on antigen presenting cells (APCs) [2]. Monoclonal proteins targeting immune checkpoints such as anti-CTLA-4 antibodies and anti-PD-1 and anti-PD-1 ligand (PD-L1 and PD-L2) antibodies have shown promising results in the treatment of solid tumors and hematological malignancies. This review will discuss the role of these antibodies as well as other immune checkpoint inhibitors (CPI) in non-Hodgkin lymphoma (NHL).

\section{Role of Tumor Microenvironment in Immune Escape}

Malignant B cells in lymphoma have the ability to evade host immune responses, and this is in part due to lymphoma cell interactions with the tumor microenvironment (TME) 


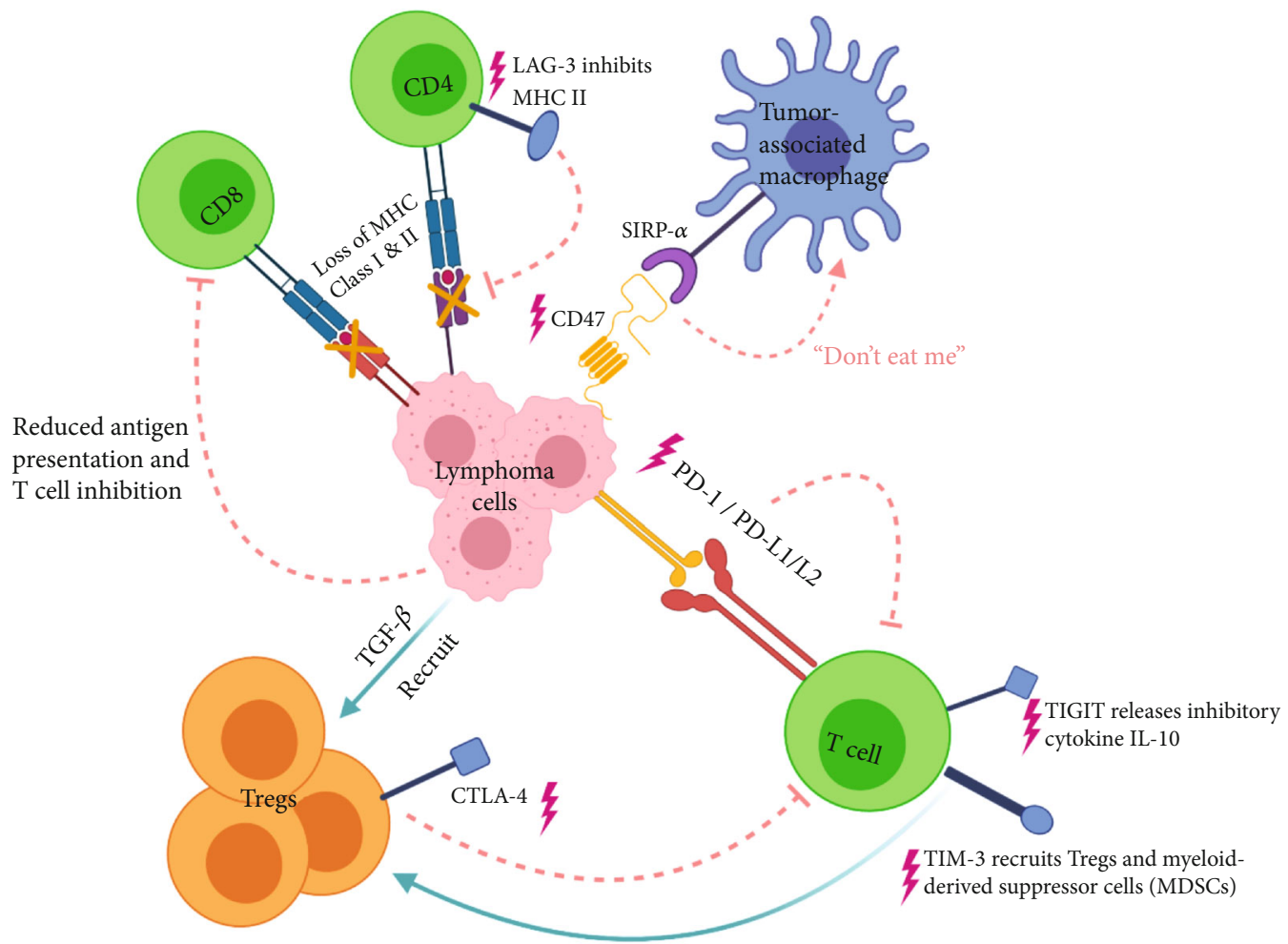

: target for CPI therapy; Tregs: regulatory T cells; SIRP- $\alpha$ : signal regulatory protein $\alpha$; TIGIT: T cell immunoglobulin and ITIM domain; TIM-3: T cell immunoglobulin and mucin domain containing protein 3; LAG-3: lymphocyte activation gene 3

FIGURE 1: Mechanisms of immune escape by lymphoma cells.

(Figure 1). The TME is complex and heterogenous and comprises of tumor cells, immune cells, stromal cells, blood vessels, and a variety of associated tissue cells. Immune cells present in the tumor include components of the innate (macrophages, dendritic cells, etc.) and adaptive immune system (B and $\mathrm{T}$ cells). $\mathrm{T}$ cell activation, which is the first step in mounting an effective immune response, occurs when antigen presenting cells (APCs) such as macrophages and dendritic cells present foreign antigens to host $\mathrm{T}$ cells. Activation of $\mathrm{T}$ cells is initiated via $\mathrm{T}$ cell receptor engagement with major histocompatibility complex (MHC) class I and II molecules on APCs. A second activating signal, typically mediated via CD28, CD27, and tumor necrosis factor receptor superfamily proteins, is required for adequate $\mathrm{T}$ cell function. An overenthusiastic T cell response is mitigated by induction of $\mathrm{T}$ cell inhibitory signals via CTLA-4, PD-1, CD160, and B and T lymphocyte-associated protein (BTLA) [3]. Tumor cells capitalize on these regulatory pathways by overexpressing inhibitory ligands or secreting immunosuppressive cytokines, thereby dampening an effective immune response [4].

An effective and appropriate immune response relies on adequate antigen presentation in the context of MHC molecules. Lymphoma cells themselves act as antigen presenting cells but are only weakly immunogenic because of reduced expression of MHC on their surface [5]. Loss of MHC occurs either due to homozygous deletion of MHC class II genes or chromosomal translocations in the $\mathrm{MHC}$ master regulator $[6,7]$, resulting in reduced presentation of tumor-associated antigens to host CD4+ T helper cells and therefore reduced activation of cytotoxic T lymphocytes (CTLs). These findings have been confirmed by DNA microarray analysis that shows fewer CTLs in the TME in MHC II negative than MHC II expressing DLBCL biopsy samples $[8,9]$. The role of MHC II can be further affected by lymphocyte activating gene 3 (LAG-3) signaling, an inhibitory coreceptor that binds to MHC class II additionally suppressing its function [10]. Furthermore, MHC loss triggers upregulation of CD47 which interacts with signal regulatory protein alpha $(\operatorname{SIRP} \alpha)$ to send a "don't eat me" signal to phagocytic cells within the TME [11].

Overexpression of inhibitory ligands on lymphoma cells can suppress an effective antitumor $\mathrm{T}$ cell response. Genetic amplification in lymphoma cells at the chromosome 9p locus and associated upregulation of the JAK2 genes results in expression of aberrant surface markers, particularly CD274 and CD273 or programmed cell death ligands 1 and 2 (PD$\mathrm{L} 1 / \mathrm{PD}-\mathrm{L} 2)$. These proteins interact with the $\mathrm{PD}-1$ receptor on CD4+ T cells and CTLs to provide inhibitory signals as a negative regulator of $\mathrm{T}$ cell activity. Uncontrolled immune activation has been documented in PD-1 and PD-L1 null mice, highlighting the importance of balance between stimulatory and inhibitory signals in T cell function $[12,13]$. In lymphoma patients, however, there may be upregulation of the PD-L1 and/or PD-L2 expression on the malignant cells that inhibits the $\mathrm{T}$ cell response and results in a state of reduced $\mathrm{T}$ cell differentiation, decreased proliferation, and suppressed effector function. Upregulation of these ligands is also seen within the TME cells, commonly on intratumoral 
macrophages. Immune checkpoints in lymphoma may be amenable to therapeutic manipulation to allow $\mathrm{T}$ cell reactivation either by blocking inhibitory interactions or promoting agonistic stimulatory signals.

Additionally, regulatory $\mathrm{T}$ cells (Tregs) that are part of the TME suppress $\mathrm{T}$ cell function and play an important role in immune homeostasis and self-tolerance by inhibiting cytotoxic $\mathrm{T}$ cells. Lymphoma cells actively recruit Tregs to the TME to suppress the antitumor immune response and promote Treg differentiation via immunosuppressive cytokines such as tumor growth factor $\beta$ (TGF- $\beta$ ). TGF- $\beta$ suppresses effector $\mathrm{T}$ cell function by causing $\mathrm{T}$ cells to differentiate into Tregs [14]. Tregs express CTLA-4 which is an inhibitory molecule that suppresses $\mathrm{T}$ cell function by assisting in Treg-mediated downregulation of stimulatory molecules CD80 and CD86 on dendritic cells. Inhibition of CTLA-4 inhibits FoxP3+ Tregs from inhibiting effector T cell function [15]. Due to signaling induced by inhibitory ligands, the secretion of immunosuppressive cytokines, and the presence of cells with regulatory function, the antitumor immune response is effectively suppressed in most lymphoma patients.

\section{Biological Basis of Checkpoint Blockade in NHL}

As mentioned above, $\mathrm{T}$ cell function is governed by stimulatory and inhibitory molecules to ensure an optimal response. Immune checkpoint proteins, which tend to be upregulated in lymphoma, send inhibitory signals to activated $\mathrm{T}$ cells and cause suppression of $\mathrm{T}$ cell function. Reactivating $\mathrm{T}$ cells forms the basis of immune checkpoint inhibitor (CPI) therapy. An ongoing understanding of $\mathrm{T}$ cell biology has prompted the development of therapies capable of restoring $\mathrm{T}$ cell function. As a strategy to prevent $\mathrm{T}$ cell suppression, checkpoint inhibitors have been developed against multiple inhibitory proteins responsible for regulating the adaptive immune system including PD-1/PD-L1, CTLA-4, TIM3, LAG3, and TIGIT and against inhibitory pathways regulating the innate immune system including CD47. Here, we discuss the rationale behind some of these therapies.

3.1. PD-1/PD-1 Ligand Signaling. The therapeutic success of immune checkpoint blockade in classical Hodgkin lymphoma ( $\mathrm{cHL}$ ) has largely been attributed to the high prevalence of 9p24.1 amplification and the enhanced PD-1/PDL1/2 expression on tumor cells and in the TME in this disease. In contrast, NHLs are more heterogenous and do not share the same biological features as $\mathrm{cHL}$, in that the PD1/PD-L1 expression in NHL has inconsistently been associated with prognosis [16]. However, there are subgroups of NHLs that share a common genetic signature, and high rates of 9p24.1 gain similar to cHL. These include primary mediastinal B cell lymphoma (PMBL), primary CNS lymphoma (PCNSL), primary testicular lymphoma (PTL), and gray zone lymphoma (GZL) [17-21], all of which have increased expression of $\mathrm{PD}-\mathrm{L} 1 / 2$ due to genetic upregulation. Relative expression of PD-L2, compared to PD-L1, is increased in PMBL with more than $70 \%$ of tumor cells expressing the ligand and polymerase chain reaction (PCR) testing confirming copy number gains in PD-L2 in these patients [17]. In patients with PCNSL and PTL, recurrent translocations of regulatory elements of TBLX1XR1 and PD-L2 gene as well as genes upstream of PD-L1 and PD-L2 were observed [18]. These tumors, in comparison to other types of NHL, have high expression of PD1, PD-L1, and/or PD-L2, which forms the basis for CPI in these conditions, and therapeutic responses have been seen in these diseases in early phase clinical trials.

In contrast, 9p24.1 copy number alterations (CNAs) resulting in the increased PD-1/PD-L1 expression are seen in only a handful of patients with de novo diffuse large B cell lymphoma (DLBCL), and the link to prognosis in these patients is not clear [22]. Burkitt lymphoma and mantle cell lymphoma cells also have virtually no PD-L1/2 expression detected on tumor cells $[16,23]$. Similarly, follicular lymphoma cells seldom have 9p24.1 gain and have inconsistent expression of PD-L1 on their surface. However, the high PD-1 expression on tumor infiltrating lymphocytes (TILs) of the TME in follicular lymphoma (FL) has been observed, and this has variably corresponded with a shorter time to progression or high-grade transformation [16, 24-27]. PD1 and LAG-3 signaling blockade resulted in CD8+ T cell function restoration in these patients [28]. In comparison, DLBCL has lower levels of PD-1 expressing TILs, and patients with DLBCL have been noted to have circulating PD-1, but the prognostic impact of these findings remains unclear [29-32]. Whether the expression of checkpoint proteins on cells from the TME in lymphoma predicts sensitivity to CPI therapy is as yet unknown.

Chronic viral infection, especially with EBV, upregulates the PD-1 [23] and PD-L1 [33] expression, and the PD-L1 expression corresponds with clinical responses to $\mathrm{PD}-1$ blockade [16]. This provides a rationale for the use of CPI in EBV-associated lymphomas such as EBV positive DLBCL, NK/T cell lymphomas, and posttransplant lymphoproliferative disorders (PTLD). PTLD in particular show high levels of both PD-1 and PD-L1 expression and may be amenable to CPI therapy.

3.2. CTLA4 Expression. CTLA-4 is an inhibitory receptor of the $\mathrm{B} 7$ family and negative regulator of $\mathrm{T}$ cell activation. It can be detected on regulatory T cells (Tregs), as well as activated CD4+ and CD8+ T cells, and acts by enhancing Treg function and dampening the immune response [15]. CTLA- 4 blockade has been shown to enhance the activity of endogenous antitumor $\mathrm{T}$ cells, thereby inducing tumor regression [34]. Anti-CTLA-4 antibodies, ipilimumab and tremelimumab, have been extensively studied in melanoma patients, and in 2011, the Federal Drug Administration (FDA) approved ipilimumab for treatment of metastatic melanoma. In patients with NHL, the efficacy of CTLA4 inhibition was modest at best, with only 2 of 18 patients showing a response in a phase 1 trial [35]. However, combination therapies that include anti-CTLA-4 antibodies and synergistic agents to prime the immune system have significantly enhanced antitumor responses, as shown in the EL4 lymphoma mouse model where coadministration of dendritic 
cell vaccine and anti-CTLA-4 antibody resulted in tumor responses in $60 \%$ of the mice, whereas neither agent alone prevented tumor growth [36]. This suggests that the efficacy of CTLA4 inhibition may require additional modulation of immune function.

3.3. Other Checkpoint Proteins. In addition to the two checkpoint molecules mentioned above, inhibitory molecules against other immune checkpoint proteins are currently being investigated. These proteins include $\mathrm{T}$ cell immunoglobulin and ITIM domain (TIGIT), T cell immunoglobulin and mucin domain containing protein 3 (TIM-3), and lymphocyte activation gene 3 (LAG-3). TIGIT interacts with the poliovirus receptor (PVR) and increases IL-10 production, which is an inhibitory cytokine $[37,38]$. Anti-TIGIT antibodies have shown some promising results in mouse models [39], and clinical trials are being designed to test them further. TIM-3 regulates T helper cells and helps with recruitment of myeloid-derived suppressor cells (MDSCs), which are potent suppressors of $\mathrm{T}$ cell immunity via production of nitric oxide (monocytic MDSCs) and pathways involving IFN- $\gamma$, production of reactive oxygen species, and arginine metabolism (granulocytic MDSCs) [40]. High expression of TIM-3 on DLBCL tumor cells was associated with a worse overall and progression free survival [41]. Trials using antiTIM-3 antibodies in relapsed lymphoma are ongoing to test safety and efficacy in human subjects. Similarly, LAG-3 interacts with CD3 to mediate inhibition of T cell proliferation and cytokine production [42]. Anti-LAG-3 antibodies are also being tested in hematological malignancies.

3.4. Targeting Regulators of the Innate Immune System. Macrophages, monocytes, and natural killer (NK) cells form part of the innate immune system and a similarly controlled by immune regulatory receptors. CD47, a regulator of the phagocytosis, is ubiquitous on all normal tissues with upregulation seen in malignant cells. The receptor for this ligand is signal regulatory protein $\alpha(\operatorname{SIRP} \alpha)$, which is present on monocytes and macrophages, dendritic cells, and granulocytes. Interaction between these proteins sends a "do noteat-me" signal to macrophages and monocytes which downregulates malignant cell phagocytosis [11, 43]. Anti-CD47 antibodies can block this inhibitory signal and promote phagocytosis, particularly antibody-mediated cellular cytotoxicity (ADCC) [44]. However, blockade of this pathway has the potential of off-target toxicity due to the universal expression of CD47 [45] that includes anemia and thrombocytopenia. Despite this, humanized anti-CD47 antibody molecules have been shown to promote macrophage-mediated phagocytosis in NHL engrafted mouse xenografts without indiscriminate killing of normal cells $[46,47]$. This has translated into clinical benefit with a phase Ib trial of anti-CD47 antibody in combination with rituximab showing a $40 \%$ response rate in DLBCL and 71\% response rate in FL [48].

Another potential therapeutic target that may regulate the innate immune system is the killer-cell immunoglobulin-like receptor (KIR) expressed on NK cells. Interaction between KIR and MHC class I molecules induces NK cell tolerance [49]. Anti-KIR antibodies have shown enhancement of NK cell-mediated cytotoxicity, an effect further augmented when combined with rituximab [50].

3.5. Combination Therapy. Single agent CPI results in responses in only a fraction of patients with non-Hodgkin lymphoma. Combination therapies using strategies that further enhance immune function are currently being explored and include improving antigen presentation, promoting $\mathrm{T}$ helper cell response, dual checkpoint blockade, and enhancing $\mathrm{T}$ cell activation. Chemotherapy and radiotherapy, which disrupt DNA, enhance expression of pattern recognition receptors (PRRs) on cells of the innate immune system thereby improving antigen presentation by APCs and macrophages $[51,52]$. This forms a basis for current trials investigating combination chemotherapy or radiotherapy with CPI in lymphomas.

Aside from the immune checkpoint combinations mentioned above, small molecule inhibitors have potential activity in combination with CPI. One agent which has shown synergy in vitro is ibrutinib, a BTK inhibitor which suppresses $B$ cell receptor (BCR) signaling that is essential for malignant B cell survival. Ibrutinib, however, also targets interleukin-2 inducible kinase (ITK) in T cells and may shift the balance between Th1 and Th2 T cells, thereby enhancing the antitumor response. Lymphoma mouse models have shown that ibrutinib can potentiate $\mathrm{T}$ cell responses in presence of PD-1 blockade causing a synergistic effect [53].

Further, mouse models show where PD-1 and TIM-3 coexpressing TILs are present; they represent a more exhausted phenotype and dual blockade of TIM-3 and PD1 may result in better tumor control [54]. Similar results were seen in an A20 lymphoma mouse model which showed coexpression of PD-1 and TIGIT, and near complete disappearance of tumor was seen when mice were treated with both anti-PD-1 and anti-TIGIT antibodies [55]. In a further mouse model, the combination of an anti-PD-1 antibody with an anti-CTLA-4 antibody resulted in an augmented antitumor response due to an increase in intratumoral cytokines [56]. These preclinical results suggest that combinations of immune checkpoint blocking antibodies may result in a more effective antilymphoma $\mathrm{T}$ cell response than the use of each CPI alone. These combinations may also include immune checkpoint inhibitors targeting both the adaptive and innate immune system. Clinical results thus far testing these combinations have been disappointing. In a phase 1 study combining PD-1 blockade (nivolumab) with either an anti-CTLA-4 antibody (ipilimumab) or anti-KIR antibody (lirilumab) resulted in an ORR of $9-22 \%$ in patients with NHL, and toxicity from the combinations was greater than that seen with the single agents alone [57]. Other clinical studies to establish the most effective combinations are currently ongoing.

\section{Clinical Efficacy of Checkpoint Inhibition}

Despite the promise of preclinical studies, the clinical efficacy of CPI in NHL has been modest as shown by a number of early phase clinical trials. In some subclasses of NHL, the response rates are as high as $40 \%-50 \%$, particularly in 
patients with a 9p24.1 amplification that results in PD-L1/2 overexpression. In other types of lymphoma, the response rates are poor. Unfortunately, a large percentage of patients with NHLs do not gain benefit from CPI therapy. Currently, there are 20 registered trials studying efficacy of checkpoint blockade as single agents or in combination with other therapies in patients with different types of NHL. A summary of select completed trials is listed in Table 1.

4.1. NHL with Upregulation of 9p24.1. Some subgroups of non-Hodgkin lymphoma which show amplification of the 9p24.1 locus respond very well to PD-1 blockade. These histologies include primary mediastinal B cell lymphoma (PMBL), gray zone lymphoma (GZL), primary CNS lymphoma (PCNSL), and primary testicular lymphoma (PTL). The KEYNOTE-170 trial, which enrolled 53 patients with relapsed/refractory PMBL, reported an ORR of $45 \%$ with a $13 \% \mathrm{CR}$ rate in pembrolizumab-treated patients. At a median follow-up of 12.5 months, the median duration of response and overall survival was not reached. At the time of the report, all patients in CR continued in $\mathrm{CR}$ including one patient off therapy for 12 months [58, 59]. Similar promising results have been observed in patients with relapsed/refractory PCNSL and PTL treated with nivolumab. In a small series of patients, all five patients (4 PCNSL and 1 PTL) responded including 4 CRs after 3 cycles of treatment. At a follow-up of 17 months, three maintained a response, and all 5 patients were alive [60]. In addition, 3 of 3 patients with gray zone lymphoma in a separate report achieved durable CR with PD-1 blockade [61].

4.2. Diffuse Large B Cell Lymphoma. Disappointingly, checkpoint inhibitors as single agent therapy have limited efficacy in diffuse large B cell lymphoma (DLBCL). In a phase 1 trial of ipilimumab, an anti-CTLA-4 antibody, in patients with relapsed or refractory NHL, only one patient with DLBCL had a durable response [35]. Results with PD-1 blockade were initially more promising. In a phase 1 study of nivolumab, an ORR of $35 \%$ was observed among patients with DLBCL; however, durability was poor with most patients relapsing by 3 months [62]. Subsequently, in the CHECKMATE-139 trial, nivolumab was administered to 121 patients with relapsed/refractory DLBCL ( $\mathrm{r} / \mathrm{r}$ DLBCL) who had either failed or were ineligible for an autologous stem cell transplant (ASCT). At a median posttreatment follow-up of 9 months in the auto-ASCT-failed cohort and 6 months in the auto-ASCT-ineligible cohort, response rates were $10 \%$ and $3 \%$, respectively. The median durations of response were less than 12 months with a median OS 12.2 months and 5.8 months, respectively. Further, only $3 \%$ of patients showed evidence of chromosome 9p24.1 amplification [63]. Similarly, pembrolizumab was studied in patients with DLBCL as consolidation post-ASCT with the goal of improving the PFS at 18 months from $60 \%$ to $80 \%$. Unfortunately, in the 29 patients enrolled, the 18.5-month PFS was $58 \%$ with increased toxicity confirming posttransplant PD1 blockade does not improve therapeutic benefit in unselected DLBCL patients.
One subgroup of large cell lymphoma that has shown some initial encouraging results is patients with Richter's transformation (RT). These patients tend to have the high PD-1 expression on the tumor cells [64], and a promising response rate to pembrolizumab therapy was demonstrated in a cohort of patients with CLL [65]. Confirmatory studies were unfortunately disappointing with most patients in the KEYNOTE-170 RT cohort progressing. Only 3 (one with DLBCL histology and 2 with cHL histology) of 23 patients responded and even those patients had poor response durability [66].

In contrast, checkpoint inhibitors targeting proteins of the innate immune system appear to have greater efficacy. Blockade of CD47/SIRP $\alpha$ signaling with TTI-621 showed an ORR of $36 \%$ and CR of $14 \%$ in $\mathrm{r} / \mathrm{r}$ DLBCL [67]. Treatment with Hu5F9-G4, an anti-CD47 antibody, plus rituximab resulted in an ORR of $40 \%$ with a CR rate of $33 \%$ in DLBCL patients [48].

Similar to single agent therapy, combination immune checkpoint therapy in unselected DLBCL patients has shown limited efficacy. Nivolumab combined with ipilimumab demonstrated an ORR of $36 \%$ in $\mathrm{r} / \mathrm{r}$ DLBCL with increased toxicity compared to single agent [68]. Further, nivolumab combined with either ipilimumab or lirilumab (anti-KIR antibody) resulted in an ORR of $19 \%$ and $13 \%$, respectively, in patients with NHL in a phase 1 trial [57]. Similarly, and despite in vitro efficacy, ibrutinib combined with PD-1 blockade resulted in an ORR of $36 \%$ only in a clinical study [69]. In select populations, however, the results are more encouraging. Thirty patients with $\mathrm{r} / \mathrm{r}$ PMBL treated with nivolumab and brentuximab vedotin in a phase 2 trial showed an ORR of $70 \%$ with $\mathrm{CR}$ of $43 \%$ [70]. These data stress the importance of appropriate patient selection in patients with lymphomas, and studies combining tumor-directed therapy and immune checkpoint blockade in selected populations are now underway.

4.3. Follicular and Other Low-Grade Lymphomas. Relapsed/refractory low-grade lymphomas can be challenging to treat due to their propensity to relapse. Although thought to be inherently immunosensitive due to response to nonspecific immune blockade, results with CPI in follicular lymphoma (FL) have been mixed. An initial phase 1 study with ipilimumab showed only a single durable response [35]. More promising results were seen with nivolumab in a phase I trial showing a $40 \%$ response rate, and most responses were durable for up to 2 years [62]. However, CHECKMATE-140, a phase 2 trial of nivolumab in relapsed/refractory FL $(r / r$ FL), showed very modest results in the enrolled 92 patients with an ORR of only $4 \%$, a median DOR of 11 months, and a PFS of only 2.2 months [71]. Success with pembrolizumab has also been limited, as seen in a phase 2 trial of low-grade lymphomas which included 23 patients: 18 with FL, 2 with marginal zone lymphoma (MZL), and 3 with lymphoplasmacytic lymphoma (LPL). Two FL patients had partial responses, and one LPL patient had a minor response, but the duration of response (DOR) was only 5.5 and 4.9 months, respectively, and the MZL patient having a $40 \%$ tumor reduction with ongoing therapy. Overall, the median PFS for all patients was only 3.4 months [72]. 


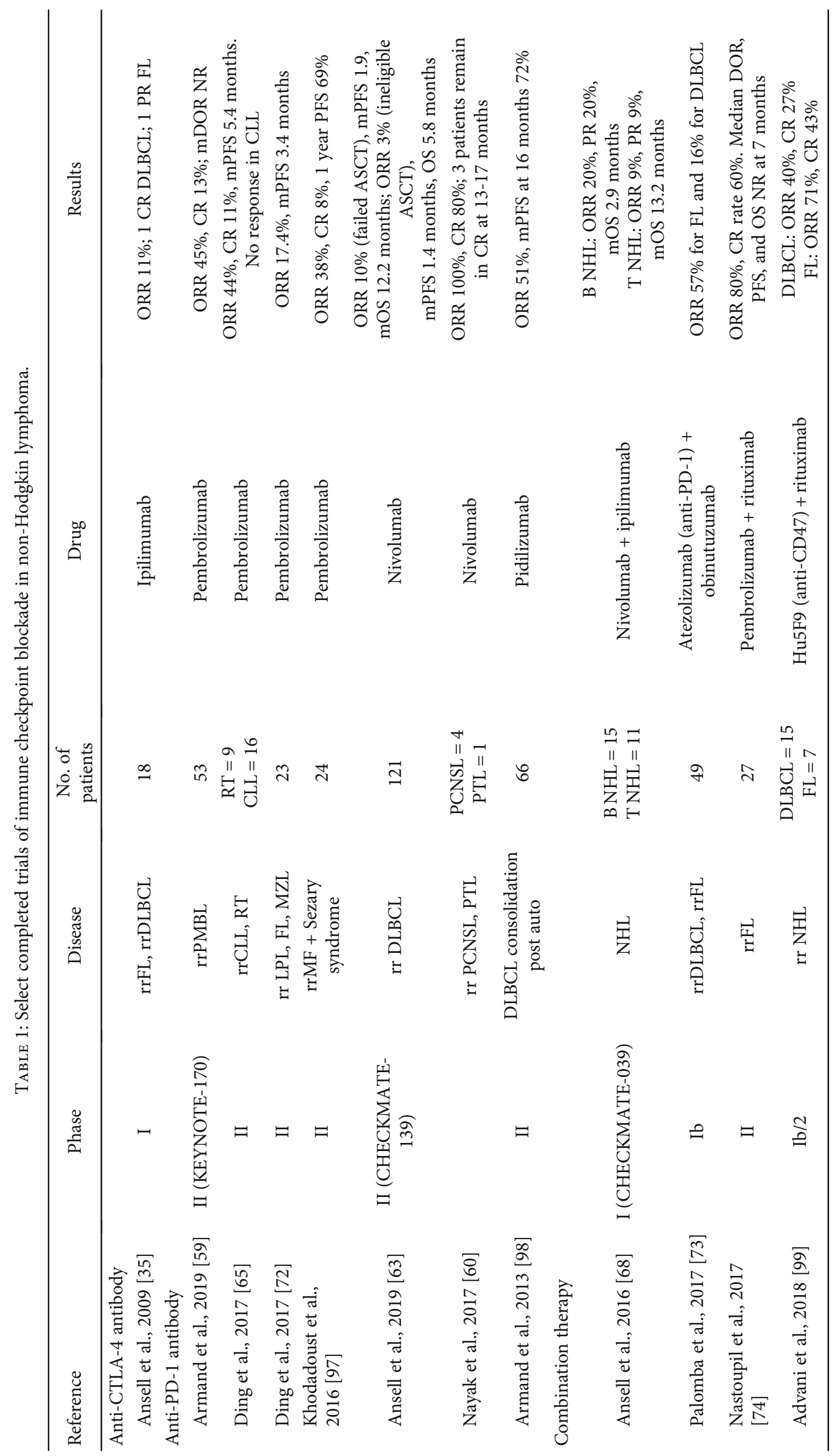


Combination therapy in FL has been more encouraging in comparison. In a phase 1 trial of atezolizumab and obinutuzumab, the ORR was $57 \%$ in $\mathrm{r} / \mathrm{r}$ FL [73], whereas a phase 2 trial of pembrolizumab with rituximab demonstrated an ORR of $80 \%$ with a CR rate of $60 \%$. The median PFS and OS in this study were not reached at 7 months [74]. Further, rituximab combined with utomilumab achieved an ORR of $33 \%$ in patients with $\mathrm{r} / \mathrm{r}$ FL who were rituximab refractory [75]. Ongoing clinical trials are now exploring combination therapies with checkpoint blockade and chemoimmunotherapy, radiotherapy, and immunomodulators.

4.4. T Cell Lymphoma. T cell lymphomas are typically a difficult group of diseases to manage, and these diseases are often associated with a poor prognosis especially in the relapse$\mathrm{d}$ /refractory setting. Response to CPI therapy is often dictated by the type of $\mathrm{T}$ cell lymphoma with some histologies responding better than others. NK/T cell lymphomas, which are often associated with EBV infection, have been shown to have an upregulation of the PD-L1 expression and tend to respond favorably to $\mathrm{PD}-1$ blockade $[76,77]$. In the $\mathrm{r} / \mathrm{r}$ setting, 5 of 7 patients in one cohort and 7 of 7 patients in a second cohort responded to PD-1 blockade. Other types of T cell lymphomas have had more mixed results. Nivolumab in $\mathrm{r} / \mathrm{r}$ PTCL and mycosis fungoides showed an ORR of $40 \%$ and $15 \%$, respectively, in a phase 1 trial [62]. A phase 2 trial of pembrolizumab enrolled 23 patients with advanced MF. The ORR was $38 \%$ with $89 \%$ of responses durable at a median of 32 weeks resulting in a one-year PFS of $69 \%$. In contrast, only one of 13 patients with PTCL achieved a response with combination therapy with nivolumab and ipilimumab [68]. Despite some studies showing reassuring results, there have been reports of hyperprogression in some cohorts on patients with PTCL. At least 2 groups of investigators have reported rapid disease progression in a subset of patients after initiation of checkpoint inhibition $[78,79]$.

4.5. Summary. Responses seen with checkpoint inhibitor therapy in NHL do not compare with the success seen in cHL. Some patients with NHL respond well to this therapy, especially those with chromosome 9p24.1 amplification, including patients with PMBL, GZL, PCNSL, and PTL. A further group of responding patients is those with EBV positive lymphomas which also tend to upregulate the PD-1/PD-L1 expression [80, 81]. Specifically, NK/T cell lymphomas which tend to be EBV driven and also show upregulation of PD-L1 have demonstrated encouraging results. Other EBV positive lymphomas such as posttransplant lymphoproliferative disorder (PTLD) which have upregulated the PD-L1 expression may also be amenable to checkpoint blockade [81].

\section{Adverse Events with Checkpoint Blockade}

As discussed, checkpoint blockade inhibits the immunosuppressive $T$ cell signal which allows tumor cells to evade the immune system and proliferate undetected. As a result, there is immune activation when these agents are administered therapeutically. Although the exact mechanism is unknown, the working hypothesis is that most side effects are due to failure of immunological tolerance resulting in $\mathrm{T}$ cellmediated immune-related adverse events (irAE) [82]. Furthermore, cytokines may also play a role as evidenced by a rise in interleukin-17 levels in patients with ipilimumabinduced colitis [83]. These events usually occur within the first few weeks or months after therapy is initiated. Delayed onset of immune side effects after therapy cessation has also been reported with waxing and waning of symptoms. Encouragingly, data from melanoma studies does not show any long-term side effects [84]. Fatal toxic events have been reported and occurred in $0.3 \%$ to $1.3 \%$ of patients treated, usually occurring early in the treatment course [85]. Immune-related AEs can affect almost any organ and may be different depending on the immune checkpoint inhibitor used, e.g., colitis and hypophysitis are more common with anti-CTLA-4 therapy, and pneumonitis and thyroiditis are more common with anti-PD-1 therapy. Overall, immune adverse events appear to be more common with CTLA-4 blockade (60\%-90\%) compared to PD-1/PD-L1 blockade (40\%-70\%), with the incidence being higher in patients receiving combination therapy $[82,86]$. Myocarditis is a rare side effect of CPI, and in hematological malignancies treated with CPI, it is almost never seen. The exact reason for this discrepancy is as yet unknown.

Many events can be managed with simple measures such as the use of anti-inflammatory or antipyretic agents including NSAIDs or antihistamines, or by stopping the offending drug. Sometimes, medications that can curb an overactive T cell response are necessary such as corticosteroids, calcineurin inhibitors, and anti-TNF $\alpha$ inhibitors like infliximab. Whether it is safe to restart CPI therapy after an irAE has occurred has not been studied in prospective trials. Retrospective analysis suggests that recurrent or new immune reactions can occur with repeat therapy [87]. While these events tend to be less severe, the decision to recommence treatment should be individualized depending on nature of the malignancy, the severity of the prior event, and whether feasible alternative treatment options are available.

A concerning feature reported in some $\mathrm{T}$ cell lymphoma trials with CPI therapy is rapid disease progression resulting in death. Twelve patients with relapsed/refractory PTCL $(\mathrm{r} / \mathrm{r}$ PTCL) were treated with nivolumab at the Mayo Clinic, and although 4 patients responded to treatment, there were 4 instances of hyperprogression, defined as dramatic disease progression within one cycle of treatment. The study was terminated early as a result [79]. Furthermore, 3 patients with adult $\mathrm{T}$ cell leukemia lymphoma (ATLL) treated with nivolumab showed rapid disease progression after one dose of the medication [78]. In addition, Zinzani et al. [88] noted 3 episodes of hyperprogression leading to death in a cohort of 44 patients with $\mathrm{r} / \mathrm{r}$ PTCL treated with Tislelizumab [88]. Careful thought therefore needs to be given to patient selection in $\mathrm{T}$ cell lymphoma trials utilizing CPI therapy.

\section{Biomarkers}

Checkpoint therapy has shown some remarkable results in early phase trials; however, not all patients respond to treatment and novel biomarkers to predict response and help 
patient selection are therefore necessary. We know from studies in solid tumors that the increased PD-1/PD-L1 expression is associated with responses to checkpoint blockade $[89,90]$. In patients with lymphoma, however, the predictive value of the PD-1/PD-L1 expression is inconsistent. Trials in Hodgkin lymphoma showed that the elevated PDL1 expression on tumor cells corresponded with a higher ORR $[91,92] . H$ scores, calculated as the number of PD-L1 positive malignant cells multiplied by the intensity of PDL1 positive staining, correlated with 9p24.1 amplification, which in turn predicted response. However, some patients with low $H$ scores also had a clinical response [93].

In contrast to Hodgkin lymphoma, the usefulness of the PD-L1 expression, detected in the TME or serum, as a biomarker in DLBCL has been less clear. Soluble PD-L1 (sPDL1) was detected in pretreatment plasma samples from an initial cohort of 283 DLBCL patients from France and a confirmatory cohort of 225 patients from North America. Both cohorts showed an inferior overall survival in patients with elevated sPD-L1. This however did not correlate with tumor or TME expression of PD-L1, and tumor PD-L1 did not predict survival in patients $[94,95]$. In a study using nivolumab in relapsed DLBCL, the prevalence of copy number gain or amplification of chromosome 9p24.1 was low, and the tumor expression of PD-L1 did not correlate with response. Of the 2 patients who had a CR, one had 9p24.1 amplification, whereas the other one had normal levels. In those with PR, none had the PD-L1 expression on tumor cells [63].

However, in a small cohort of patients with Richter's transformation treated with pembrolizumab, the increased PD-L1 expression was detected in patients with a confirmed response. Other markers tested included chromosome 9p24.1 alterations, EBV status, and MSI status, but none of these correlated with treatment response in CLL patients with RT [65]. In the KEYNOTE-170 trial of pembrolizumab in relapsed PMBL, the tumor $H$ score was calculated for PDL1. A rise in $H$ score signifying higher expression of PD-L1 and amplification of 9p24.1 gain strongly correlated with response to therapy, and the $\mathrm{PD}-\mathrm{L} 1$ expression was also strongly associated with progression free survival [59].

In a study of patients with follicular lymphoma, the higher expression of PD-L1 was seen at baseline on peripheral blood $\mathrm{T}$ cells among responders when treated with pidilizumab [96]. Further, the increased expression of a T cell activation gene signature in pretreatment tumor samples was associated with a longer PFS in patients treated with PD-1 blockade. This was not seen in a separate study where patients were treated with chemotherapy alone [96]. This suggests that the gene signature may be useful in predicting outcomes in patients post PD-1 blockade therapy, but larger studies with higher patient numbers are needed to confirm this finding.

\section{Conclusion}

Checkpoint inhibitors disrupt the inhibitory feedback loop of the immune system rather than target tumor cells. Treatment with these agents has shown promising results in some relapsed and refractory non-Hodgkin lymphomas, which continue to be an area of unmet need. However, appropriate patient selection appears to be the key in determining treatment outcome, as some histologies of NHL respond well to CPI while many others do not. As discussed in this review, patients with primary mediastinal B cell lymphoma, primary CNS lymphoma, testicular lymphoma, and gray zone lymphoma are most likely to respond favorably. The PD-L1 expression has been shown to predict response in some studies, but this is inconsistent, and a reliable biomarker to predict response is currently lacking. Moving forward, newer ICP targets will need to be considered. Potential molecules including TIM-3, LAG-3, TIGIT, and BTLA have shown encouraging results in preclinical models, and clinical trials exploring their efficacy are currently accruing patients. Agents targeting the innate immune system are also being investigated, and anti-CD47 and anti-KIR antibodies appear to hold promise in early studies. Furthermore, combination therapy with more than one CPI or CPI with other targeted agents and/or chemotherapy is being investigated. All told, we anticipate that rational combination approaches that include immunological agents will be necessary to improve the outcome of patients with NHL.

\section{Conflicts of Interest}

The authors declare that there is no conflict of interest regarding the publication of this paper.

\section{References}

[1] A. M. Lesokhin, M. K. Callahan, M. A. Postow, and J. D. Wolchok, "On being less tolerant : enhanced cancer immunosurveillance enabled by targeting checkpoints and agonists of $\mathrm{T}$ cell activation," Science Translational Medicine, vol. 7, pp. 113, 2015.

[2] J. F. Grosso and M. N. Jure-kunkel, "CTLA-4 blockade in tumor models : an overview of preclinical and translational research," Cancer Immunity, vol. 13, pp. 1-14, 2013.

[3] S. M. Ansell, "Harnessing the power of the immune system in non-Hodgkin lymphoma: immunomodulators, checkpoint inhibitors, and beyond," Hematology, vol. 2017, no. 1, pp. 618-621, 2017.

[4] Z.-Z. Yang, A.-B. Liang, and S. M. Ansell, “T-cell-mediated antitumor immunity in B-cell non-Hodgkin lymphoma: activation, suppression and exhaustion," Leukemia \& Lymphoma, vol. 56, no. 9, pp. 2498-2504, 2015.

[5] M. Nijland, R. N. Veenstra, L. Visser et al., "HLA dependent immune escape mechanisms in B-cell lymphomas : implications for immune checkpoint inhibitor therapy ?," OncoImmunology, vol. 6, no. 4, pp. e1295202-e1295208, 2017.

[6] S. A. Riemersma, E. S. Jordanova, R. F. J. Schop et al., "Extensive genetic alterations of the HLA region, including homozygous deletions of HLA class II genes in B-cell lymphomas arising in immune-privileged sites," Blood, vol. 96, no. 10, pp. 3569-3577, 2000.

[7] C. Steidl, S. P. Shah, B. W. Woolcock et al., "MHC class II transactivator CIITA is a recurrent gene fusion partner in lymphoid cancers," Nature, vol. 471, no. 7338, pp. 377-381, 2011.

[8] L. M. Rimsza, R. A. Roberts, T. P. Miller et al., "Loss of MHC class II gene and protein expression in diffuse large B-cell 
lymphoma is related to decreased tumor immunosurveillance and poor patient survival regardless of other prognostic factors: a follow-up study from the Leukemia and Lymphoma Molecular Profiling Project," Blood, vol. 103, no. 11, pp. 4251-4258, 2004.

[9] D. W. Scott and R. D. Gascoyne, "The tumour microenvironment in B cell lymphomas," Nature Reviews Cancer, vol. 14, no. 8, pp. 517-534, 2014.

[10] T. Maruhashi, I. M. Okazaki, D. Sugiura et al., "LAG-3 inhibits the activation of CD4(+) T cells that recognize stable pMHCII through its conformation-dependent recognition of pMHCII," Nature Immunology, vol. 19, no. 12, pp. 1415-1426, 2018.

[11] A. N. Barclay, K. Timo, and D. Berg, "The interaction between signal regulatory protein alpha (SIRP $\alpha$ ) and CD47: Structure, Function, and therapeutic target," Annual Review of Immunology, vol. 32, no. 1, pp. 25-50, 2014.

[12] H. Nishimura, N. Minato, T. Nakano, and T. Honjo, "Immunological studies on PD-1 deficient mice: implication of PD1 as a negative regulator for B cell responses," International Immunology, vol. 10, no. 10, pp. 1563-1572, 1998.

[13] H. Nishimura, M. Nose, H. Hiai, N. Minato, and T. Honjo, "Development of lupus-like autoimmune diseases by disruption of the PD-1 gene encoding an ITIM motif-carrying immunoreceptor," Immunity, vol. 11, no. 2, pp. 141-151, 1999.

[14] Z. Yang, D. M. Grote, S. C. Ziesmer et al., "Soluble and membrane-bound TGF- $\beta$-Mediated regulation of intratumoral $\mathrm{T}$ cell differentiation and function in B-cell nonHodgkin lymphoma," PLoS One, vol. 8, no. 3, pp. 1-13, 2013.

[15] K. Wing, Y. Onishi, P. Prieto-Martin et al., "CTLA-4 control over Foxp3 + regulatory T cell function," Science, vol. 322, no. 5899, pp. 271-275, 2008.

[16] Z. Y. Xu-Monette, J. Zhou, and K. H. Young, "PD-1 expression and clinical PD-1 blockade in B-cell lymphomas," Blood, vol. 131, no. 1, pp. 68-83, 2018.

[17] M. Shi, M. G. M. Roemer, B. Chapuy et al., "Expression of programmed cell death 1 ligand 2 (PD-L2) is a distinguishing feature of primary mediastinal (thymic) large B-cell lymphoma and associated with PDCD1LG2 copy gain," The American Journal of Surgical Pathology, vol. 38, no. 12, pp. 1715-1723, 2014.

[18] B. Chapuy, M. G. M. Roemer, C. Stewart et al., "Targetable genetic features of primary testicular and primary central nervous system lymphomas," Blood, vol. 127, no. 7, pp. 869-881, 2016.

[19] D. D. W. Twa, F. C. Chan, S. Ben-Neriah et al., "Genomic rearrangements involving programmed death ligands are recurrent in primary mediastinal large B-cell lymphoma," Blood, vol. 123, no. 13, pp. 2062-2065, 2014.

[20] R. W. Merryman, P. Armand, K. T. Wright, and S. J. Rodig, "Checkpoint blockade in Hodgkin and non-Hodgkin lymphoma," Blood Advances, vol. 1, no. 26, pp. 2643-2654, 2017.

[21] F. C. Eberle, I. Salaverria, C. Steidl et al., "Gray zone lymphoma: chromosomal aberrations with immunophenotypic and clinical correlations," Modern Pathology, vol. 24, no. 12, pp. 1586-1597, 2011.

[22] D. Kwon, S. Kim, P. J. Kim et al., "Clinicopathological analysis of programmed cell death 1 and programmed cell death ligand 1 expression in the tumour microenvironments of diffuse large B cell lymphomas," Histopathology, vol. 68, no. 7, pp. 10791089, 2016.
[23] B. J. Chen, B. Chapuy, J. Ouyang et al., "PD-L1 expression is characteristic of a subset of aggressive B-cell lymphomas and virus-associated malignancies," Clinical Cancer Research, vol. 19, no. 13, pp. 3462-3473, 2013.

[24] Z. Yang, D. M. Grote, S. C. Ziesmer, B. Xiu, A. J. Novak, and S. M. Ansell, "PD-1 expression defines two distinct T-cell sub-populations in follicular lymphoma that differentially impact patient survival," Blood Cancer Journal, vol. 5, no. 2, pp. e281-e281, 2015.

[25] J. P. Smeltzer, J. M. Jones, S. C. Ziesmer et al., "Pattern of CD14+ follicular dendritic cells and PD1+ T cells independently predicts time to transformation in follicular lymphoma," Clinical Cancer Research, vol. 20, no. 11, pp. 2862-2872, 2014.

[26] J. Carreras, A. Lopez-Guillermo, G. Roncador et al., "High numbers of tumor-infiltrating programmed cell death 1-positive regulatory lymphocytes are associated with improved overall survival in follicular lymphoma," Journal of Clinical Oncology, vol. 27, no. 9, pp. 1470-1476, 2009.

[27] S. Muenst, S. Hoeller, N. Willi, S. Dirnhofer, and A. Tzankov, "Diagnostic and prognostic utility of PD-1 in B cell lymphomas," Disease Markers, vol. 29, no. 1, 53 pages, 2010.

[28] Z.-Z. Yang, H. J. Kim, J. C. Villasboas et al., "Expression of LAG-3 defines exhaustion of intratumoral PD-1 + T cells and correlates with poor outcome in follicular lymphoma," Oncotarget, vol. 8, no. 37, pp. 61425-61439, 2017.

[29] M. J. Ahearne, K. Bhuller, R. Hew, H. Ibrahim, K. Naresh, and S. D. Wagner, "Expression of PD-1 ( CD279) and FoxP3 in diffuse large B-cell lymphoma," Virchows Archiv, vol. 465, no. 3, pp. 351-358, 2014.

[30] J. Kiyasu, H. Miyoshi, A. Hirata et al., "Expression of programmed cell death ligand 1 is associated with poor overall survival in patients with diffuse large B-cell lymphoma," Blood, vol. 126, no. 19, pp. 2193-2201, 2015.

[31] M. Cohen, A. G. Vistarop, F. Huaman et al., "Cytotoxic response against Epstein Barr virus coexists with diffuse large B-cell lymphoma tolerogenic microenvironment : clinical features and survival impact," Nature, vol. 7, no. 1, pp. 1-10, 2017.

[32] W. Zhang, J. F. Bai, M. X. Zuo et al., "PD-1 expression on the surface of peripheral blood CD4+T cell and its association with the prognosis of patients with diffuse large B-cell lymphoma," Cancer Medicine, vol. 5, no. 11, pp. 30773084, 2016.

[33] M. R. Green, S. Rodig, P. Juszczynski et al., "Constitutive AP-1 activity and EBV infection induce PD-L1 in Hodgkin lymphomas and posttransplant lymphoproliferative disorders: implications for targeted therapy," Clinical Cancer Research, vol. 18, no. 6, pp. 1611-1618, 2012.

[34] D. R. Leach, M. F. Krummel, and J. P. Allison, "Enhancement of antitumor immunity by CTLA-4 blockade," Science, vol. 271, no. 5256, pp. 1734-1736, 1996.

[35] S. M. Ansell, S. A. Hurvitz, P. A. Koenig et al., "Cancer Therapy : Clinical phase I study of ipilimumab , an anti - CTLA-4 monoclonal antibody, in patients with relapsed and refractory B-cell non - Hodgkin lymphoma," Clinical Cancer Research, vol. 4, pp. 6446-6454, 2009.

[36] O. Met, M. Wang, A. E. Pedersen, and M. H. Nissen, "The effect of a therapeutic dendritic cell-based cancer vaccination depends on the blockage of CTLA-4 signaling," Cancer Letters, vol. 231, no. 2, pp. 247-256, 2006. 
[37] N. Stanietsky, H. Simic, J. Arapovic et al., "The interaction of TIGIT with PVR and PVRL2 inhibits human NK cell cytotoxicity," Proceedings of the National Academy of Sciences, vol. 106, no. 42, pp. 17858-17863, 2009.

[38] X. Yu, K. Harden, L. C Gonzalez et al., "The surface protein TIGIT suppresses $\mathrm{T}$ cell activation by promoting the generation of mature immunoregulatory dendritic cells," Nature Immunology, vol. 10, no. 1, pp. 48-57, 2009.

[39] K. O. Dixon, M. Schorer, J. Nevin et al., "Functional antiTIGIT antibodies regulate development of autoimmunity and antitumor immunity," The Journal of Immunology, vol. 200, no. 8, pp. 3000-3007, 2018.

[40] V. Dardalhon, A. C. Anderson, J. Karman et al., “Tim-3/Galectin-9 pathway: regulation of Th1 immunity through promotion of CD11b + Ly-6G + myeloid cells," Journal of Immunology, vol. 185, no. 3, pp. 1383-1392, 2010.

[41] B. J. Chen, R. Dashnamoorthy, P. Galera et al., "The immune checkpoint molecules PD-1, PD-L1, TIM-3 and LAG-3 in diffuse large B-cell lymphoma," Oncotarget, vol. 10, no. 21, pp. 2030-2040, 2019.

[42] S. Hannier, M. Tournier, G. Bismuth, and F. Triebel, "CD3/TCR complex-associated lymphocyte activation gene-3 molecules inhibit CD3/TCR signaling," Journal of Immunology, vol. 161, pp. 4058-4065, 1998.

[43] S. Jaiswal, C. H. M. Jamieson, W. W. Pang et al., "CD47 is upregulated on circulating hematopoietic stem cells and leukemia cells to avoid phagocytosis," Cell, vol. 138, no. 2, pp. 271285, 2009.

[44] R. Majeti, M. P. Chao, A. A. Alizadeh et al., "CD47 is an adverse prognostic factor and therapeutic antibody target on human acute myeloid leukemia stem cells," Cell, vol. 138, no. 2, pp. 286-299, 2009.

[45] X. W. Zhao, T. W. Kuijpers, and T. K. van den Berg, "Is targeting of CD47-SIRP $\alpha$ enough for treating hematopoietic malignancy?," Blood, vol. 119, no. 18, pp. 4333-4334, 2012.

[46] M. P. Chao, A. A. Alizadeh, C. Tang et al., "Anti-CD47 antibody synergizes with rituximab to promote phagocytosis and eradicate non-Hodgkin lymphoma," Cell, vol. 142, no. 5, pp. 699-713, 2010.

[47] P. S. Petrova, N. N. Viller, M. Wong et al., "TTI-621 (SIRP $\alpha$ Fc): a CD47-blocking innate immune checkpoint inhibitor with broad antitumor activity and minimal erythrocyte binding," Clinical Cancer Research, vol. 23, no. 4, pp. 1068-1079, 2017.

[48] R. Advani, I. Flinn, L. Popplewell et al., "CD47 blockade by Hu5F9-G4 and rituximab in non-Hodgkin's lymphoma," The New England Journal of Medicine, vol. 379, no. 18, pp. 17111721, 2018.

[49] A. Thielens, E. Vivier, and F. Romagné, "NK cell MHC class I specific receptors (KIR): from biology to clinical intervention," Current Opinion in Immunology, vol. 24, no. 2, pp. 239-245, 2012.

[50] H. E. Kohrt, A. Thielens, A. Marabelle et al., "Anti-KIR antibody enhancement of anti-lymphoma activity of natural killer cells as monotherapy and in combination with anti-CD20 antibodies," Blood, vol. 123, no. 5, pp. 678686, 2014.

[51] O. Takeuchi and S. Akira, "Pattern recognition receptors and inflammation," Cell, vol. 140, no. 6, pp. 805-820, 2010.

[52] S. A. Patel and A. J. Minn, "Combination cancer therapy with immune checkpoint blockade: mechanisms and strategies," Immunity, vol. 48, no. 3, pp. 417-433, 2018.
[53] I. Sagiv-Barfi, H. E. K. Kohrt, D. K. Czerwinski, P. P. Ng, B. Y. Chang, and R. Levy, "Therapeutic antitumor immunity by checkpoint blockade is enhanced by ibrutinib, an inhibitor of both BTK and ITK," Proceedings of the National Academy of Sciences, vol. 112, no. 9, pp. E966-E972, 2015.

[54] K. Sakuishi, L. Apetoh, J. M. Sullivan, B. R. Blazar, V. K. Kuchroo, and A. C. Anderson, "Targeting Tim-3 and PD-1 pathways to reverse $\mathrm{T}$ cell exhaustion and restore anti-tumor immunity," The Journal of Experimental Medicine, vol. 207, no. 10, pp. 2187-2194, 2010.

[55] N. Sunseri, X. Chen, N. Wald et al., "Beyond PD-1: investigating the therapeutic potential of TIGIT blockade in DLBCL," Blood, vol. 134, Supplement_1, p. 391, 2019.

[56] M. Selby, J. Engelhardt, L. S. Lu et al., "Antitumor activity of concurrent blockade of immune checkpoint molecules CTLA-4 and PD-1 in preclinical models," Journal of Clinical Oncology, vol. 31, 15_suppl, p. 3061, 2013.

[57] P. Armand, A. Lesokhin, I. Borrello et al., "A phase 1b study of dual PD- 1 and CTLA-4 or KIR blockade in patients with relapsed / refractory lymphoid malignancies," Leukemia, 2020.

[58] P. L. Zinzani, V. Ribrag, C. H. Moskowitz et al., "Safety and tolerability of pembrolizumab in patients with relapsed/refractory primary mediastinal large B-cell lymphoma," Blood, vol. 130, no. 3, pp. 267-270, 2017.

[59] P. Armand, S. Rodig, V. Melnichenko et al., "Pembrolizumab in relapsed or refractory primary mediastinal large B-cell lymphoma," Journal of Clinical Oncology, vol. 37, no. 34, pp. 32913299, 2019.

[60] L. Nayak, F. M. Iwamoto, A. LaCasce et al., "PD-1 blockade with nivolumab in relapsed/refractory primary central nervous system and testicular lymphoma," Blood, vol. 129, no. 23, pp. 3071-3073, 2017.

[61] C. Melani, A. Major, J. Schowinsky et al., "PD-1 blockade in mediastinal gray-zone lymphoma," The New England Journal of Medicine, vol. 377, no. 1, pp. 89-91, 2017.

[62] A. M. Lesokhin, S. M. Ansell, P. Armand et al., "Nivolumab in patients with relapsed or refractory hematologic malignancy: preliminary results of a phase Ib study," Journal of Clinical Oncology, vol. 34, no. 23, pp. 2698-2704, 2016.

[63] S. M. Ansell, M. C. Minnema, P. Johnson et al., "Nivolumab for relapsed/refractory diffuse large B-cell lymphoma in patients ineligible for or having failed autologous transplantation: a single-arm, phase II study," Journal of Clinical Oncology, vol. 37, no. 6, pp. 481-489, 2019.

[64] R. He, W. Ding, D. S. Viswanatha et al., "PD-1 expression in chronic lymphocytic leukemia/small lymphocytic lymphoma (CLL/SLL) and large B-cell Richter transformation (DLBCLRT): a characteristic feature of DLBCL-RT and potential surrogate marker for clonal relatedness," The American Journal of Surgical Pathology, vol. 42, no. 7, pp. 843-854, 2018.

[65] W. Ding, B. R. LaPlant, T. G. Call et al., "Pembrolizumab in patients with CLL and Richter transformation or with relapsed CLL," Blood, vol. 129, no. 26, pp. 3419-3427, 2017.

[66] P. Armand, N. Murawski, D. Molin et al., "Pembrolizumab in relapsed or refractory Richter syndrome," British Journal of Haematology, vol. 190, no. 2, pp. e117-e120, 2020.

[67] S. M. Ansell, I. W. Flinn, M. B. Maris et al., "TTI-621 (SIR$\mathrm{P} \alpha \mathrm{Fc}$ ), an immune checkpoint inhibitor blocking the CD47 "do not eat" signal, induces objective responses in patients with advanced, relapsed/refractory diffuse large B-cell lymphoma (DLBCL)," Blood, vol. 130, p. 4116, 2017. 
[68] S. Ansell, M. E. Gutierrez, M. A. Shipp et al., "A phase 1 study of nivolumab in combination with ipilimumab for relapsed or refractory hematologic malignancies (CheckMate 039)," Blood, vol. 128, no. 22, p. 183, 2016.

[69] A. Younes, J. Brody, C. Carpio et al., "Safety and activity of ibrutinib in combination with nivolumab in patients with relapsed non-Hodgkin lymphoma or chronic lymphocytic leukaemia: a phase 1/2a study," The Lancet Haematolog, vol. 6, no. 2, pp. e67-e78, 2019.

[70] P. L. Zinzani, A. Santoro, G. Gritti et al., "Nivolumab combined with brentuximab vedotin for relapsed/refractory primary mediastinal large B-cell lymphoma: efficacy and safety from the phase II CheckMate 436 study," Journal of Clinical Oncology: Official Journal of the American Society of Clinical Oncology, vol. 37, no. 33, pp. 3081-3089, 2019.

[71] P. Armand, A. M. H. Janssens, G. Gritti et al., "Efficacy and safety results from CheckMate 140, a phase 2 study of nivolumab for relapsed/refractory follicular lymphoma," Blood, 2020.

[72] W. Ding, B. Laplant, T. E. Witzig et al., "PD-1 blockade with pembrolizumab in relapsed low grade non-Hodgkin lymphoma," Blood, vol. 130, p. 4055, 2017.

[73] M. L. Palomba, B. G. Till, S. I. Park et al., "A phase IB study evaluating the safety and clinical activity of atezolizumab combined with obinutuzumab in patients with relapsed or refractory non-Hodgkin lymphoma (NHL)," Hematological Oncology, vol. 35, pp. 137-138, 2017.

[74] L. J. Nastoupil, J. Westin, N. Fowler et al., "High response rates with pembrolizumab in combination with rituximab in patients with relapsed follicular lymphoma: interim results of an ON open-label, phase II study," Hematological Oncology, vol. 35, pp. 120-121, 2017.

[75] A. Gopal, R. Levy, R. Houot et al., “A phase I study of utomilumab (PF-05082566), a 4-1BB/CD137 agonist, in combination with rituximab in patients with CD20+non-Hodgkin's lymphoma," Hematological Oncology, vol. 35, p. 260, 2017.

[76] Y.-L. Kwong, T. S. Y. Chan, D. Tan et al., "PD1 blockade with pembrolizumab is highly effective in relapsed or refractory NK/T-cell lymphoma failing l-asparaginase," Blood, vol. 129, no. 17, pp. 2437-2442, 2017.

[77] X. Li, Y. Cheng, M. Zhang et al., "Activity of pembrolizumab in relapsed/refractory NK/T-cell lymphoma," Journal of Hematology \& Oncology, vol. 11, no. 1, p. 15, 2018.

[78] L. Ratner, T. A. Waldmann, M. Janakiram, and J. E. Brammer, "Rapid progression of adult T-cell leukemia-lymphoma after PD-1 inhibitor therapy," The New England Journal of Medicine, vol. 378, no. 20, pp. 1947-1948, 2018.

[79] N. N. Bennani, L. D. Pederson, P. Atherton et al., "A phase II study of nivolumab in patients with relapsed or refractory peripheral T-cell lymphoma," Blood, vol. 134, Supplement_1, p. $467,2019$.

[80] A. Carbone, A. Gloghini, and C. Carlo-Stella, "Are EBVrelated and EBV-unrelated Hodgkin lymphomas different with regard to susceptibility to checkpoint blockade?," Blood, vol. 132, no. 1, pp. 17-22, 2018.

[81] A. Kinch, C. Sundström, E. Baecklund, C. Backlin, D. Molin, and G. Enblad, "Expression of PD-1, PD-L1, and PD-L2 in posttransplant lymphoproliferative disorder after solid organ transplantation," Leukemia \& Lymphoma, vol. 60, no. 2, pp. 376-384, 2019.

[82] M. A. Postow, R. Sidlow, and M. D. Hellmann, "Immunerelated adverse events associated with immune checkpoint blockade," The New England Journal of Medicine, vol. 378, no. 2, pp. 158-168, 2018.

[83] M. K. Callahan, A. Yang, S. Tandon et al., "Evaluation of serum IL-17 levels during ipilimumab therapy: correlation with colitis,” Journal of Clinical Oncology, vol. 29, 15_suppl, p. 2505, 2011.

[84] S. L. Topalian, M. Sznol, D. F. McDermott et al., "Survival, durable tumor remission, and long-term safety in patients with advanced melanoma receiving nivolumab," Journal of Clinical Oncology: Official Journal of the American Society of Clinical Oncology, vol. 32, no. 10, pp. 1020-1030, 2014.

[85] D. Y. Wang, J. E. Salem, J. V. Cohen et al., "Fatal toxic effects associated with immune checkpoint inhibitors a systematic review and meta-analysis," JAMA Oncology, vol. 4, no. 12, pp. 1721-1728, 2018.

[86] M. Davies, "Acute and long-term adverse events associated with checkpoint blockade," Seminars in Oncology Nursing, vol. 35, no. 5, p. 150926, 2019.

[87] F. C. Santini, H. Rizvi, A. J. Plodkowski et al., "Safety and efficacy of re-treating with immunotherapy after immune-related adverse events in patients with NSCLC," Cancer Immunology Research, vol. 6, no. 9, pp. 1093-1099, 2018.

[88] P. L. Zinzani, Q. Zhang, G. Gritti et al., "Tislelizumab (BGBA317) for relapsed / refractory peripheral T cell lymphoma: safety and efficacy results from a phase 2 study," EHA Haematol., 2020.

[89] D. E. Meyers and S. Banerji, "Biomarkers of immune checkpoint inhibitor efficacy in cancer," Current Oncology, vol. 27, no. S2, pp. S106-S114, 2020.

[90] K. Buder-Bakhaya and J. C. Hassel, "Biomarkers for clinical benefit of immune checkpoint inhibitor treatment-a review from the melanoma perspective and beyond," Frontiers in Immunology, vol. 9, 2018.

[91] P. Armand, M. A. Shipp, V. Ribrag et al., "Programmed death1 blockade with pembrolizumab in patients with classical Hodgkin lymphoma after brentuximab vedotin failure," Journal of Clinical Oncology, vol. 34, no. 31, pp. 37333739, 2016.

[92] S. M. Ansell, A. M. Lesokhin, I. Borrello et al., "PD-1 blockade with nivolumab in relapsed or refractory Hodgkin's lymphoma," The New England Journal of Medicine, vol. 372, no. 4, pp. 311-319, 2015.

[93] A. Younes, A. Santoro, M. Shipp et al., "Nivolumab for classical Hodgkin's lymphoma after failure of both autologous stemcell transplantation and brentuximab vedotin: a multicentre, multicohort, single-arm phase 2 trial," The Lancet Oncology, vol. 17, no. 9, pp. 1283-1294, 2016.

[94] for the Groupe Ouest-Est des Leucémies et Autres Maladies du Sang, D. Rossille, M. Gressier et al., "High level of soluble programmed cell death ligand 1 in blood impacts overall survival in aggressive diffuse large B-cell lymphoma: results from a French multicenter clinical trial," Leukemia, vol. 28, no. 12, pp. 2367-2375, 2014.

[95] D. Rossille, I. Azzaoui, A. L. Feldman et al., "Soluble programmed death-ligand 1 as a prognostic biomarker for overall survival in patients with diffuse large B-cell lymphoma : a replication study and combined analysis of 508 patients," Leukemia, vol. 31, no. 4, pp. 988-991, 2017.

[96] J. R. Westin, F. Chu, M. Zhang et al., "Safety and activity of PD1 blockade by pidilizumab in combination with rituximab in patients with relapsed follicular lymphoma : a single group, 
open-label, phase 2 trial," The Lancet Oncology, vol. 15, no. 1, pp. 69-77, 2014.

[97] M. Khodadoust, A. H. Rook, P. Porcu et al., "Pembrolizumab for treatment of relapsed/refractory mycosis fungoides and Sezary syndrome: clinical efficacy in a CITN multicenter phase 2 study," Blood, vol. 128, no. 22, p. 181, 2016.

[98] P. Armand, A. Nagler, E. A. Weller et al., "Disabling immune tolerance by programmed death-1 blockade with pidilizumab after autologous hematopoietic stem-cell transplantation for diffuse large B-cell lymphoma: results of an international phase II trial," Journal of Clinical Oncology, vol. 31, no. 33, pp. 4199-4206, 2013.

[99] R. H. Advani, I. Flinn, L. Popplewell et al., "Activity and tolerabilty of the first-in-class anti-CD47 antibody Hu5F9-G4 with rituximab tolerated in relapsed/refractory non-Hodgkin lymphoma: initial phase 1b/2 results," Journal of Clinical Oncology, vol. 36, 15_suppl, p. 7504, 2018. 